\title{
Cristalización y Plastificación de Margarina Industrial para Panificación
}

\author{
YOLANDA SALAS SOTAMINGA $a^{*}$, IVÁN TAPIAa, MARCELO GARZÓN ${ }^{b}$ \\ a. Facultad de Ciencias Químicas, Universidad Central del Ecuador, Quito - Ecuador \\ b. Investigación y Desarrollo, Industrial Danec S.A., Sangolquí \\ *Correspondencia: salasyola@hotmail.com
}

Recibido: 15 diciembre 2010 / Aceptado: 17 julio 2011

\section{Resumen}

La margarina es una de las materias primas utilizadas en la elaboración del pan, producto de consumo diario para alrededor de 13 millones de habitantes del Ecuador. Tomando en cuenta que la calidad del pan depende de la margarina, y ésta a su vez de las estructuras cristalinas $\beta^{\prime}$ que proporcionan a la margarina consistencia homogénea, brillo y color, se estudió la influencia de los parámetros de producción estándar en las características de la margarina. Once condiciones de producción con cuatro variables: caudal, temperatura de emulsión, temperatura de enfriamiento y velocidad de cristalización, se investigaron y las diferencias estructurales se analizaron utilizando microscopia de luz polarizada, análisis reológico, análisis calorimétrico diferencial y evaluación sensorial. Los parámetros óptimos de producción que influyen directamente en la formación de estructuras cristalinas $B^{\prime}$, fueron: temperatura de enfriamiento de $14^{\circ} \mathrm{C}$ a $16^{\circ} \mathrm{C}$, caudal de emulsión de $1590 \mathrm{Kg} / \mathrm{h}$ a 1690 $\mathrm{Kg} / \mathrm{h}$, velocidad de cristalización de 220 a 240 rpm. La temperatura de emulsión fue un parámetro que no influyó en la formación de los cristales.

Palabras clave: análisis de alimentos - margarina - aceite de palma - aceite de soya.

\section{Crystallization and Plasticity of Industrial Margarine for Bakery}

\section{Abstract}

The margarine is a raw material used in the preparation of bread; a product consumed daily by around 13 million of people in Ecuador. Considering that the bread's quality depends on margarine, and this characteristic is a consequence of the presence of $\beta^{\prime}$-crystals, which give the margarine an homogeneous consistence, shine and color, we investigated the influence of the standard production parameters on the margarine's characteristics. Flow, emulsion temperature, cooling temperature and crystallization speed were tested, and the structural differences were analyzed using Polarized Light Microscopy, rheological analysis, Differential Scanning Calorimetry (DSC) and sensorial evaluation. The optimal parameters found for the formation of $B^{\prime}$ - crystals were: flow 1590 $1690 \mathrm{~kg} / \mathrm{h}$, cooling temperature $14-16^{\circ} \mathrm{C}$ and crystallization speed $220-240 \mathrm{rmp}$. The emulsion temperature was a parameter that no influenced the formation of crystals.

Key words: food analysis - margarine - palm oil - soybean oil. 


\section{Introducción}

La margarina fue desarrollada en el año 1869 por $\mathrm{H}$. Mège Mouriés, quien patentó un procedimiento para obtener una grasa untable (plástica) a partir del sebo de vacuno, que podía utilizarse como sustituto de la mantequilla, que era cara y escasa. El nombre de "margarina" se propuso a causa de la creencia de que en el sebo predominaba el ácido margárico.[2]

Hoy en día, se producen grandes cantidades y tipos de margarinas; pero, con diferentes condiciones. Con el avance de la ciencia y de la tecnología se ha logrado obtener materia prima de origen vegetal que sustituye casi en su totalidad a la grasa animal en la elaboración de margarina; pero, se incrementaron las dificultades para mantener la calidad, haciendo que cada industria cree parámetros de control de calidad.

Este estudio está dedicado a investigar los factores que influyen en la calidad de la margarina para panificación como son la presencia de grumos que ocasionan la falta de brillo y color, que se considera son consecuencia de una mala plastificación y cristalización.

\subsection{Margarina}

Es una emulsión de agua en aceite, cuya estabilidad se consigue elevando la viscosidad de la fase continua como consecuencia de una cristalización parcial mediante emulsionantes.

Las materias primas para la margarina son aceites, grasas vegetales o animales, cuyas características se modifican por fraccionamiento y transesterificación.

La margarina está compuesta de: $80 \%$ de grasa y $18 \%$ de agua estabilizada con mono y diglicéridos (aprox. el $0.5 \%$ ), lecitina bruta (aprox. el $0.25 \%$ ). Para tipos de consumo doméstico con mayor valor nutritivo se añade leche descremada o en polvo; otros componentes son: sustancias aromáticas naturales o sintéticas (diacetilo, ácido butírico), sal común, vitaminas $\mathrm{A}$ y $\mathrm{D}_{2}$; la coloración se la realiza con ß-caroteno o aceite de palma sin blanquear.

La margarina se obtiene en instalaciones de producción continua en tres fases: emulsión de la fase acuosa en la fase oleosa; enfriamiento de la emulsión y tratamiento mecánico; cristalización, conservando el tipo de emulsión y eliminación del calor de cristalización liberado.[2]

\subsection{Plasticidad}

Es la propiedad de comportarse como sólidos resistiendo a la acción de pequeñas fuerzas y, en cambio, ser dúctiles y fluir como un líquido cuando se aplica una fuerza de deformación superior a un valor límite mínimo.

Las condiciones esenciales para que exista plasticidad son:

a) Estar constituido por dos fases.

b) La fase sólida debe estar finamente dispersa.

c) Debe haber la debida proporción entre las dos fases.[3]

Las grasas para repostería tienen la apariencia de sólidos blandos más o menos homogéneos, y observadas al microscopio aparecen como una masa de pequeños cristales, empapada de una gran cantidad de aceite líquido. Tienen la estructura característica de un sólido plástico (gráfico 1); pero, con frecuencia sufren fenómenos de tixotropía (gráfico 2) cuando se aplica sobre ellas trabajo mecánico y se hacen temporalmente más suaves, no se precisa su causa; pero, puede ser el resultado de una ligera deformación de las partículas cristalinas durante el trabajo, una tendencia de los cristales a unirse y crecer, o una orientación de los cristales cuando se someten a fuerzas de deslizamiento.[3]
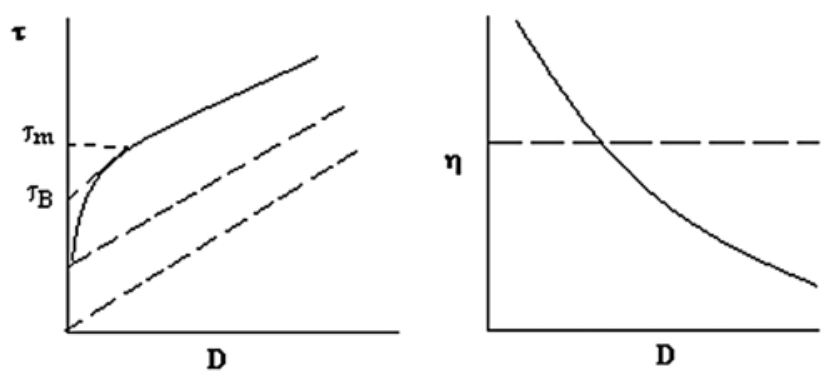

Gráfico 1. Conducta reológica del sistema plástico. [4] $\tau$ : esfuerzo cortante, D: gradiente de deslizamiento y $\eta$ : viscosidad. 

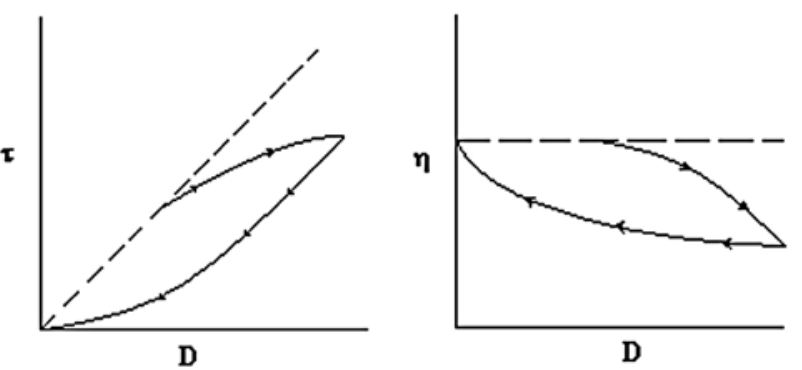

Gráfico 2. Conducta reológica del sistema tixotrópico.

[4] $\tau$ : esfuerzo cortante, $D$ : gradiente de deslizamiento y $\eta$ : viscosidad.

\subsection{Cristalización}

Es el proceso de enfriamiento de mezclas de lípidos que conduce a la formación de cristales líquidos que conforman una grasa sólida.

El tipo de cristal aparece de acuerdo a la conformación molecular de los ácidos grasos, cantidad de sólidos grasos y condiciones de enfriamiento. [2], [5], [6] y [7]

La forma cristalina primaria de los triglicéridos son a, $\beta^{\prime}$ y $\beta$ que corresponden a los principales arreglos de las cadenas de ácidos grasos (ver gráfico 3), [8].
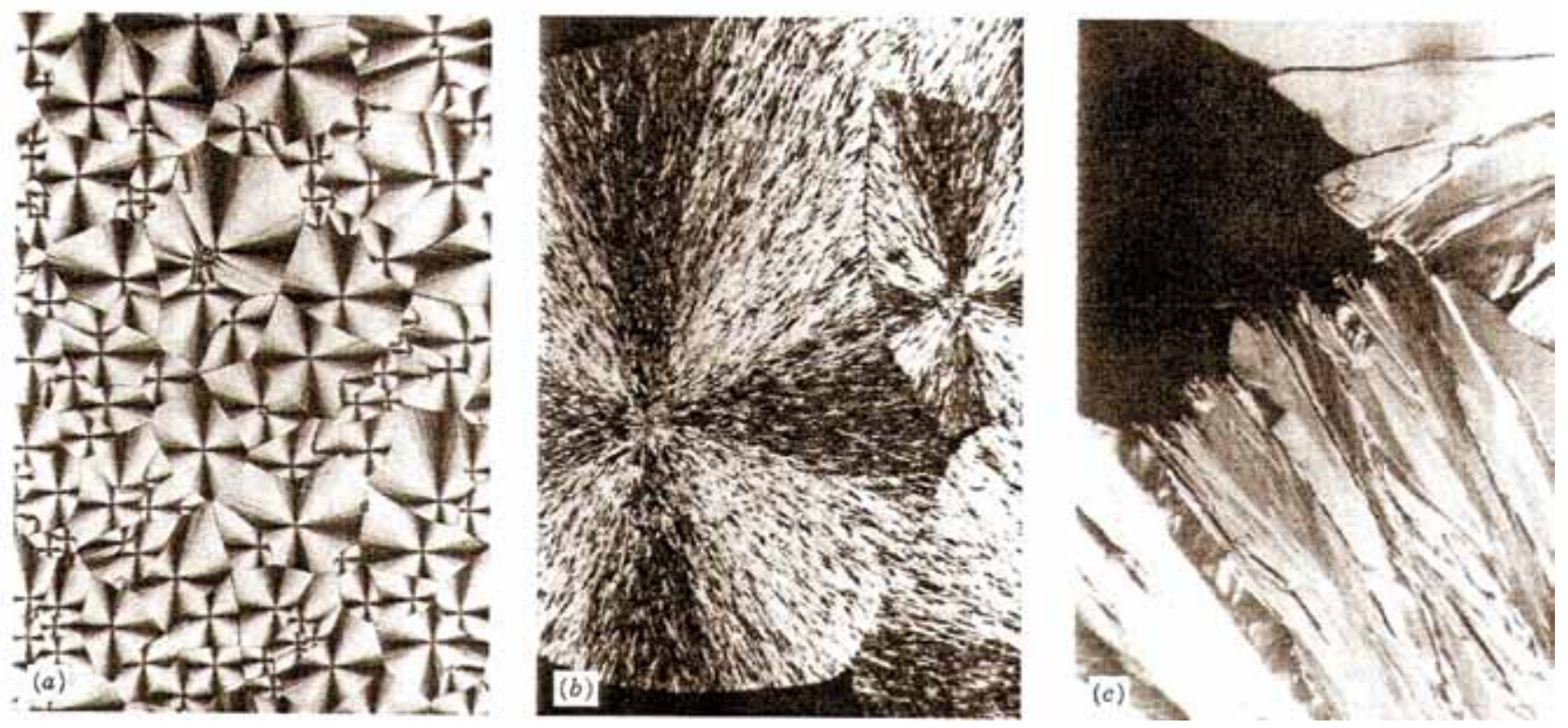

Gráfico 3. Microfotografías de cristales de grasa tomadas con luz polarizada: Derecha a izquierda; a) cristales $a$, b) cristales $B^{\prime}$, c) cristales $B^{[8]}$

Existen transformaciones entre los cristales líquidos que se dirigen a la forma polimórfica más estable $a \rightarrow$ $B \rightarrow B^{\prime}$; estos cambios son irreversibles excepto por una fusión y recristalización.[5]

A continuación se mencionan algunas características de los cristales:

B (Hexagonal).- Menos estable, bajo punto de fusión, aparece inicialmente durante condiciones de rápido enfriamiento, se transforma a la forma $\beta^{\prime}$ relativamente más estable. Tamaño cerca de $5 \mu \mathrm{m}$.
B' (Ortorrómbico Perpendicular).- Pequeños cristales como agujas juntos dentro de una densa estructura cristalina. Tamaño cerca de $1 \mu \mathrm{m}$.

B (Triclínico Paralelo).- Aparece en condiciones extremas de almacenamiento, son cristales largos rústicos estables y alto punto de fusión, el tamaño varía de 25-50 $\mu \mathrm{m}$ ○ $100 \mu \mathrm{m}$. [8], [9] y[10].

Los cristales son diferenciados por difracción de rayos $X$ y transición de calor observado en estudios calorimétricos, en los cuales se determina la conducta de 
fusión de las margarinas graficando la energía en función de la temperatura, cuya curva presenta un pico fino sobre los $4 \circ 5^{\circ} \mathrm{C}$ del punto de ablandamiento, lo que indica productos cristalizados como polimorfos $\beta^{\prime}$, mientras que la configuración $\beta$ no presenta picos en ese intervalo. [8]

Margarinas con estructuras cristalinas $B$, no poseen propiedades plásticas, ya que la red cristalina es muy resistente y al estar expuesta a una fuerza de deformación se destruye, a menudo presentan la deficiencia llamada "oiling out". A diferencia de margarinas con estructura cristalina $B^{\prime}$ de apariencia plástica que tienen la propiedad de reestablecer la red. [5]

\section{Materiales y Métodos}

\subsection{Análisis de la materia prima}

Se trabajó con muestras de aceite de palma, palmiste, soya, estearina de palma y fase oleosa.

\subsubsection{Determinación del punto de fusión}

Método: Deslizamiento [1 11$]$
Se utilizó un termómetro digital EXTECH 39240 y una fuente calorífica CORNING PC 420 D.

\subsubsection{Determinación del índice de yodo}

\section{Método: Wijs [1 1 ]}

El índice de yodo (IY) se calculó con la fórmula:

$$
\begin{aligned}
& I . Y .=\frac{\left(V_{b}-V_{m}\right) * 1.269}{p m} \\
& V_{b}=\text { Volumen gastado del blanco } \\
& V_{m}=\text { Volumen gastado de la muestra } \\
& N_{\mathrm{Na}_{2} \mathrm{~S}_{2} \mathrm{O}_{3}}=0.1 \mathrm{~N}
\end{aligned}
$$

\subsubsection{Determinación del contenido de sólidos grasos}

Método: Resonancia magnética nuclear [1 1 ] Se utilizó el equipo THE MINISPEC mq 20 NMR Analyzar Bruker y Tubos para RMN (15 cm. x 0.5cm).

\subsection{Elaboración de la margarina}

La combinación de las variables que se emplearon en los diferentes tratamientos se detalla en la tabla 1 .

Tabla 1. Combinación de variables para cada tratamiento

\begin{tabular}{cccccc}
\hline Trat. & $\begin{array}{c}\text { Temperatura de } \\
\text { emulsión } \\
\left({ }^{\circ} \mathbf{C}\right)\end{array}$ & $\begin{array}{c}\text { Temperatura de } \\
\text { enfriamiento } \\
\left({ }^{\circ} \mathbf{C}\right)\end{array}$ & $\begin{array}{c}\text { Caudal } \\
(\mathbf{k g} / \mathbf{h})\end{array}$ & \multicolumn{2}{c}{$\begin{array}{c}\text { Velocidad de } \\
\text { cristalización } \\
(\mathbf{r p m})\end{array}$} \\
\hline 1 & $47-49$ & $16-17$ & $1630-1650$ & $210-220$ & $290-310$ \\
2 & $49.5-51.5$ & $16-17$ & $1630-1650$ & $210-220$ & $290-310$ \\
3 & $52-54$ & $16-17$ & $1630-1650$ & $210-220$ & $290-310$ \\
4 & $49.5-51.5$ & $14-16$ & $1630-1650$ & $210-220$ & $290-310$ \\
5 & $49.5-51.5$ & $18-19$ & $1630-1650$ & $210-220$ & $290-310$ \\
6 & $49.5-51.5$ & $16-17$ & $1590-1610$ & $210-220$ & $290-310$ \\
7 & $49.5-51.5$ & $16-17$ & $1670-1690$ & $210-220$ & $290-310$ \\
8 & $49.5-51.5$ & $16-17$ & $1630-1650$ & $190-210$ & $290-310$ \\
9 & $49.5-51.5$ & $16-17$ & $1630-1650$ & $220-240$ & $290-310$ \\
10 & $49.5-51.5$ & $16-17$ & $1630-1650$ & $210-220$ & $270-290$ \\
11 & $49.5-51.5$ & $16-17$ & $1630-1650$ & $210-220$ & $310-330$ \\
\hline
\end{tabular}

\subsubsection{Aceptabilidad de la margarina}

Método: Sensorial [12]

El análisis se realizó con jueces no entrenados (panaderos) quienes elaboraron pan utilizando como insu- mo la margarina. Cada juez comparó la muestra con la de referencia, las características evaluadas fueron: olor, consistencia, untabilidad, aroma del pan y aspecto graso del pan. 
2.2.2. Determinación del tipo de cristal de la margarina

Método: Microscópico [13]

Se trabajó con un microscopio de luz polarizada CARL ZEISS JENA.

2.2.3. Determinación del coeficiente de deslizamiento, esfuerzo de cizalla y viscosidad

Método: Reológico

Se utilizó un Rheotest $2 \mathrm{mlw}$

\subsubsection{Determinación de las zonas de cristalización y} zonas de fusión de la margarina

Método: Calorimetría diferencial de barrido [8]
Se analizó en un calorímetro diferencial SHIMADZU DSC-50.

\section{Resultados y Discusión}

\subsection{Análisis de las materias primas}

Los datos fueron evaluados estadísticamente por comparación de medias con diseño completamente al azar, empleando la prueba $F$ (una cola) y prueba $t$ (dos colas).

\subsubsection{Punto de fusión e índice de yodo}

El punto de fusión y el índice de yodo de las diferentes materias primas estadísticamente no presentan diferencias como se observa en la tabla 2 .

Tabla 2: Resultados del ANOVA

\begin{tabular}{|c|c|c|c|c|c|c|c|c|}
\hline \multirow{3}{*}{ Muestra } & \multicolumn{4}{|c|}{ PUNTO DE FUSIÓN º } & \multicolumn{4}{|c|}{ ÍNDICE DE YODO g/100g } \\
\hline & \multicolumn{2}{|c|}{ Media Muestras } & \multirow{2}{*}{$\begin{array}{c}\text { Prueba } \\
\text { Fc* }\end{array}$} & \multirow{2}{*}{$\begin{array}{c}\text { Prueba } \\
\text { tc }^{\star \star}\end{array}$} & \multicolumn{2}{|c|}{ Media Muestras } & \multirow{2}{*}{$\begin{array}{c}\text { Prueba } \\
\text { Fc* }^{*}\end{array}$} & \multirow{2}{*}{$\begin{array}{c}\text { Prueba } \\
\text { tc }^{\star \star}\end{array}$} \\
\hline & A & B & & & A & B & & \\
\hline Aceite de palma & 33,75 & 34,75 & 1,0 & $-2,83$ & 56,05 & 53,53 & 0,64 & 7,87 \\
\hline Estearina de palma & 49,75 & 48,85 & 0,51 & 2,09 & 38,92 & 41,75 & 0,55 & $-7,00$ \\
\hline Aceite de palmaste & 28,25 & 27,05 & 25,0 & 4,71 & 18,41 & 19,73 & 1,57 & $-9,62$ \\
\hline Aceite de soya & - & - & - & - & 129,18 & 129,69 & 0,02 & $-7,63$ \\
\hline (F. oleosa) & 36,75 & 35,60 & 6,25 & 4,27 & 54,45 & 54,33 & 0,63 & 0,71 \\
\hline
\end{tabular}

${ }^{*} \mathrm{~F}_{\mathrm{T}} 95 \% 164,45$

${ }^{* *} \mathrm{t}_{\mathrm{T}} 99 \% 9,92$

\subsubsection{Contenido de sólidos grasos}

Los valores obtenidos por resonancia magnética nuclear son resultados directos del \% SFC (solid fat con- tent). La tabla 3 presenta el resumen del análisis estadístico en el cual se comprueba que existe diferencia significativa entre las materias primas estearina de palma y por ende en la mezcla.

Tabla 3: Resultados del ANOVA del contenido de sólidos grasos a $25^{\circ} \mathrm{C}$

\begin{tabular}{|c|c|c|c|c|}
\hline \multirow[b]{2}{*}{ Muestra } & \multicolumn{2}{|c|}{$\begin{array}{c}\text { Media de las muestras } \\
\%\end{array}$} & \multirow{2}{*}{$\begin{array}{l}\text { Prueba } \\
\text { Fc* }^{\star}\end{array}$} & \multirow{2}{*}{$\begin{array}{c}\text { Prueba } \\
\text { tc }^{\star \star}\end{array}$} \\
\hline & A & B & & \\
\hline Aceite de palma & 14,580 & 15,643 & 2,014 & $-6,933$ \\
\hline Estearina de palma & 52,481 & 50,441 & 6,876 & 32,315 \\
\hline Aceite de palmiste & 21,335 & $22 ., 451$ & 0,952 & $-5,584$ \\
\hline Mezcla (fase oleosa) & 16.082 & 17,623 & 8,905 & $-15,549$ \\
\hline
\end{tabular}




\subsection{Análisis de la margarina}

\subsubsection{Análisis sensorial de la margarina}

Los datos obtenidos de la evaluación sensorial (Tabla 4) fueron tratados por análisis de varianza cuyos resultados se presentan en la tabla 5 .

Tabla 4. Datos de las muestras evaluadas

\begin{tabular}{|c|c|c|c|c|c|c|c|c|c|c|c|c|c|c|c|c|c|c|}
\hline \multirow[t]{2}{*}{ Trat. } & \multicolumn{3}{|c|}{ Olor } & \multicolumn{3}{|c|}{ Consistencia } & \multicolumn{3}{|c|}{ Untabilidad } & \multicolumn{3}{|c|}{ Aceitosidad } & \multicolumn{3}{|c|}{ Aroma del pan } & \multicolumn{3}{|c|}{ Aspecto graso del pan } \\
\hline & R1 & $\mathbf{R 2}$ & $\mathbf{x}$ & R1 & $\mathbf{R 2}$ & $\mathbf{X}$ & R1 & $\mathbf{R 2}$ & $\mathbf{X}$ & R1 & $\mathbf{R 2}$ & $\mathbf{X}$ & R1 & $\mathbf{R 2}$ & $\mathbf{X}$ & $\mathbf{R 1}$ & $\mathbf{R 2}$ & $\mathbf{X}$ \\
\hline 1 & 5 & 4 & 4,5 & 4 & 6 & 5,0 & 6 & 5 & 5,5 & 4 & 4 & 4,0 & 5 & 3 & 4,0 & 5 & 4 & 4,5 \\
\hline 2 & 5 & 4 & 4,5 & 4 & 6 & 5,0 & 6 & 5 & 5,5 & 4 & 4 & 4,0 & 7 & 5 & 6,0 & 6 & 4 & 5,0 \\
\hline 3 & 5 & 4 & 4,5 & 4 & 6 & 5,0 & 3 & 5 & 4,0 & 6 & 5 & 5,5 & 2 & 5 & 3,5 & 1 & 5 & 3,0 \\
\hline 4 & 5 & 5 & 5,0 & 6 & 6 & 6,0 & 6 & 4 & 5,0 & 4 & 4 & 4,0 & 7 & 4 & 5,5 & 5 & 6 & 5,5 \\
\hline 5 & 5 & 5 & 5,0 & 5 & 6 & 5,5 & 5 & 4 & 4,5 & 4 & 4 & 4,0 & 6 & 4 & 5,0 & 6 & 5 & 5,5 \\
\hline 6 & 5 & 4 & 4,5 & 4 & 6 & 5,0 & 5 & 4 & 4,5 & 4 & 3 & 3,5 & 3 & 5 & 4,0 & 2 & 5 & 3,5 \\
\hline 7 & 4 & 4 & 4,0 & 5 & 5 & 5,0 & 4 & 4 & 4,0 & 4 & 4 & 4,0 & 5 & 5 & 5,0 & 5 & 4 & 4,5 \\
\hline 8 & 5 & 4 & 4,5 & 5 & 6 & 5,5 & 5 & 5 & 5,0 & 4 & 4 & 4,0 & 5 & 4 & 4,5 & 5 & 5 & 5,0 \\
\hline 9 & 5 & 4 & 4,5 & 5 & 6 & 5,5 & 5 & 5 & 5,0 & 4 & 4 & 4,0 & 6 & 5 & 5,5 & 6 & 5 & 5,5 \\
\hline 10 & 5 & 4 & 4,5 & 5 & 6 & 5,5 & 5 & 4 & 4,5 & 4 & 4 & 4,0 & 5 & 4 & 4,5 & 5 & 4 & 4,5 \\
\hline 11 & 5 & 4 & 4,5 & 5 & 6 & 5,5 & 5 & 5 & 5,0 & 4 & 4 & 4,0 & 5 & 3 & 4,0 & 5 & 4 & 4,5 \\
\hline
\end{tabular}

$\mathrm{R}=$ Repetición

$\mathrm{X}=$ Promedio

Tabla 5. Resumen de los resultados estadísticos del análisis sensorial

\begin{tabular}{|c|c|c|c|c|c|c|}
\hline \multicolumn{7}{|c|}{ Características } \\
\hline $\begin{array}{l}\text { Fuente de } \\
\text { Variación }\end{array}$ & 흠 & 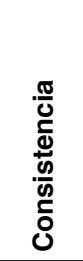 & 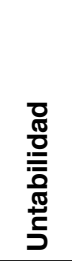 & 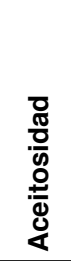 & 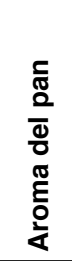 & 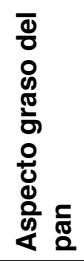 \\
\hline Muestras Fc* & 1,33 & 0,81 & 1,00 & 5,89 & 0,73 & 0,73 \\
\hline Jueces $\mathrm{Fc}^{\star \star}$ & 26,67 & 27,26 & 2,12 & 2,22 & 2,19 & 0,00 \\
\hline
\end{tabular}

${ }^{\star} \mathrm{F}_{\mathrm{T}}$ muestras $99 \% 4,85$

${ }^{\star \star} \mathrm{F}_{\mathrm{T}}$ jueces $99 \% 10.04$

De acuerdo a la comparación del $\mathrm{F}$ calculado y tabulado, la característica de la margarina descartada fue la aceitosidad; empleando la prueba de Tukey se verificó que el tratamiento 11 cuya variable fue la velocidad de cristalización, es diferente a los demás que fueron aceptados.

\subsubsection{Tipo de cristal de la margarina, resultados de microscopía}

En los gráficos 4 y 5 se presentan las fotografías tomadas de los cristales líquidos de las margarinas, utilizando un microscopio de luz polarizada con un aumento de $50 \times 0.8$.

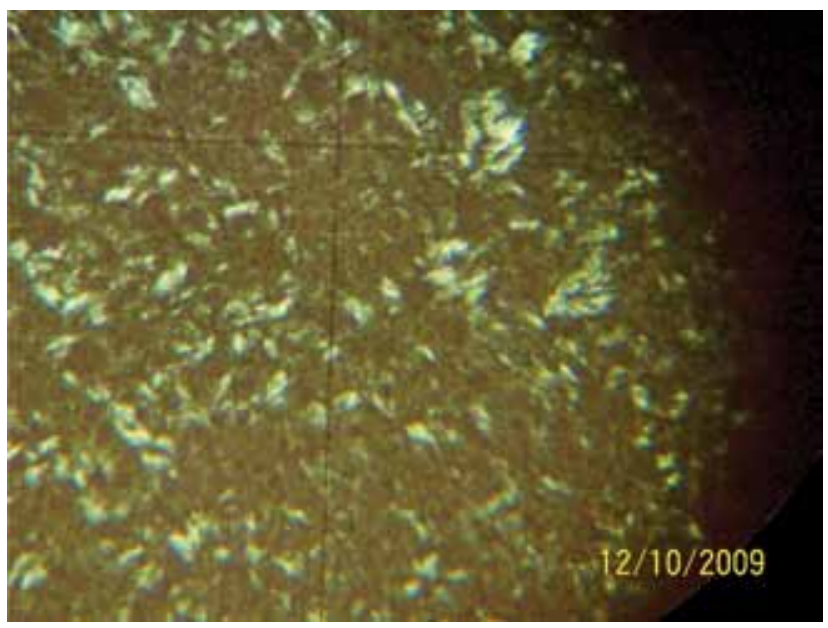

Gráfico 4. Microfotografía de cristales $\beta^{\prime}$ de margarina, tratamiento 3 


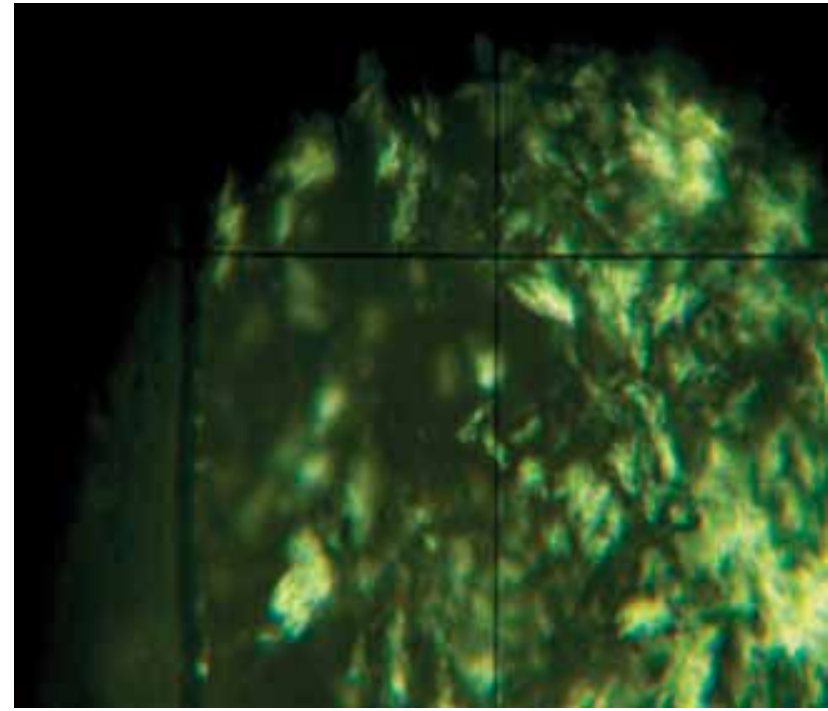

Gráfico 5. Microfotografía de cristales $\beta$ de margarina, tratamiento 6

En base al gráfico 3 se agrupó los tratamientos según el tipo de cristal y los resultados constan en la tabla 6 .

Tabla 6. Tipo de cristal de los tratamientos

\begin{tabular}{cc}
\hline Tipo de cristal & Tratamientos \\
\hline$\beta$ & $6,9,11$ \\
$\beta^{\prime}$ & $1,2,3,4,5,7,8,10$ \\
\hline
\end{tabular}

\subsubsection{Calorimetría diferencial de barrido}

El análisis se realizó a seis muestras de margarina para determinar estructuras cristalinas de forma $\beta^{\prime}$ que confirmen los resultados anteriores. Para el análisis de los termogramas se tomó en cuenta la presencia de picos sobre los $5^{\circ} \mathrm{C}$ del punto de ablandamiento.

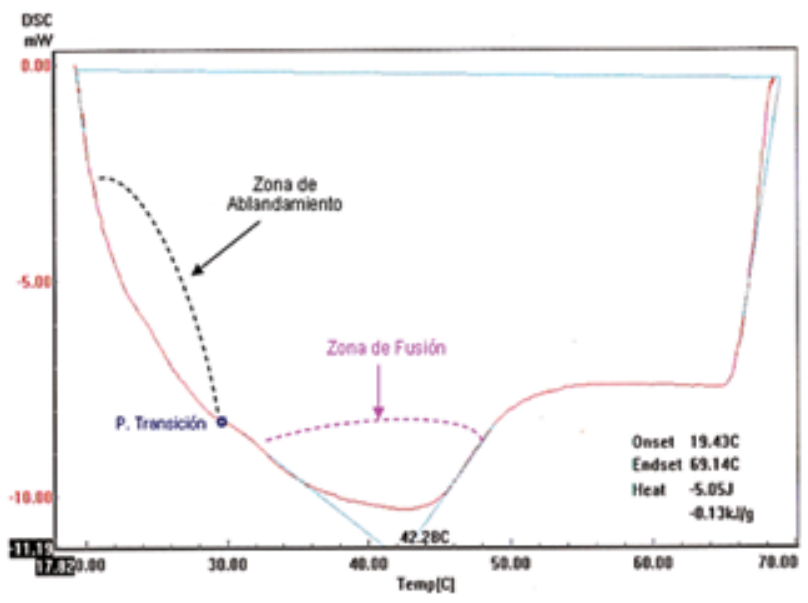

Gráfico 6. Termograma, Tratamiento 3 no existen picos, presenta cristales $B$

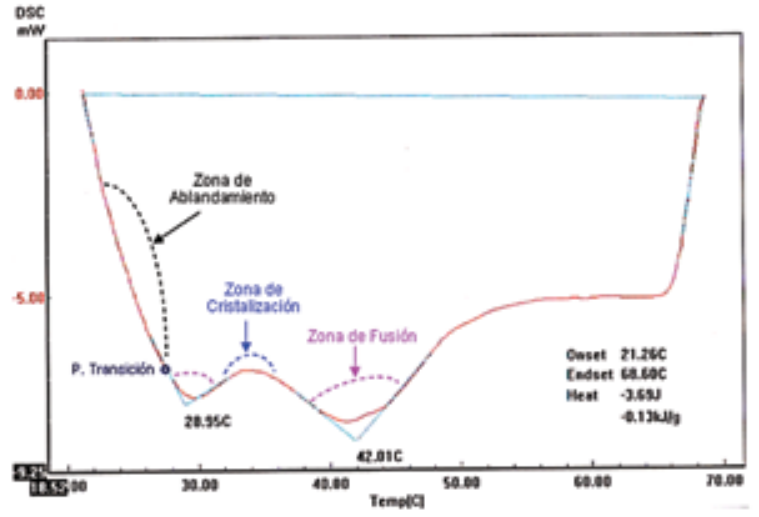

Gráfico 7. Termograma, Tratamiento 7 estructura cristalina $\beta^{\prime}$

El análisis de los termogramas condujo a los resultados de la tabla 7. Cabe señalar que el tratamiento 3 no presenta zona de cristalización, por lo tanto, existen únicamente cristales $B$.

Tabla 7. Resultados de los termogramas de calorimetría diferencial

\begin{tabular}{ccc}
\hline Trat. & Cristal & $\begin{array}{c}\text { Punto de fusión } \\
{ }^{\circ} \mathbf{C}\end{array}$ \\
\hline 1 & $\beta^{\prime}$ & 40,24 \\
3 & $\beta$ & 42,28 \\
4 & $\beta^{\prime}$ & 40.07 \\
6 & $\beta^{\prime}$ & 41.27 \\
7 & $\beta^{\prime}$ & 42.01 \\
9 & $\beta^{\prime}$ & 42.64 \\
\hline
\end{tabular}

\subsubsection{Resultados del análisis reológico}

Las curvas experimentales se analizaron en base a las curvas teóricas (Gráficos 1 y 2) para establecer similitudes y así comprobar cuál margarina tiene características plásticas.

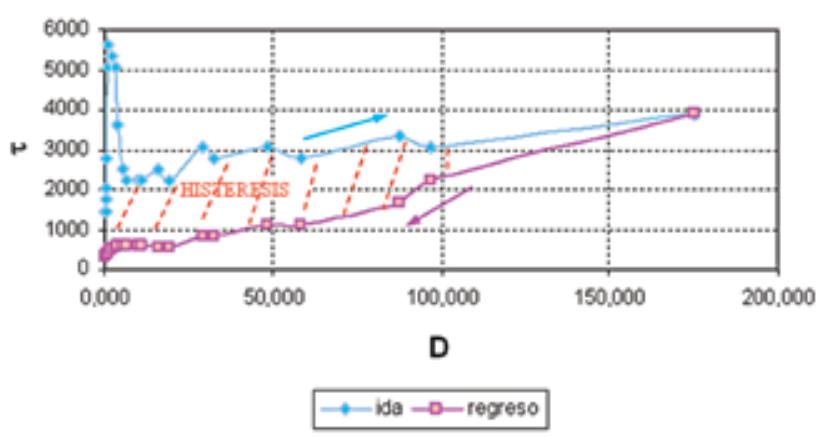

Gráfico 8. Reología: Curva del esfuerzo cortante vs. gradiente de deslizamiento, Tratamiento 4 


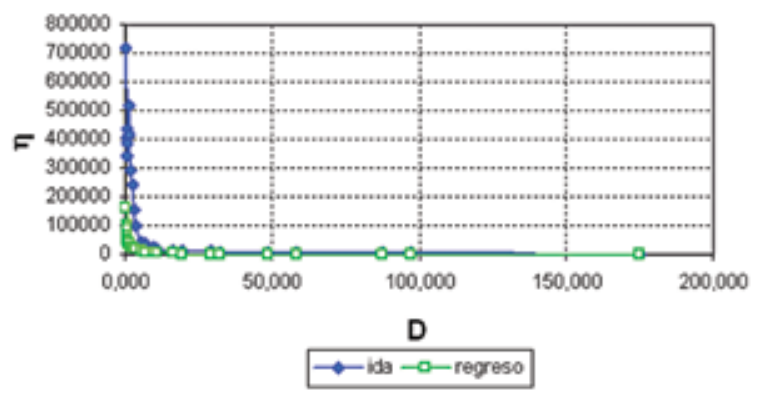

Gráfico 9: Reología: Curva de viscosidad vs. gradiente de deslizamiento. Tratamiento 4

Tabla 8. Resultados de las curvas de reología

\begin{tabular}{|c|c|c|c|c|}
\hline \multirow{3}{*}{ Trat. } & \multirow{3}{*}{ Sistema } & \multicolumn{3}{|c|}{ Valores de las curvas $T-D$} \\
\hline & & \multirow{2}{*}{$\begin{array}{c}\text { Punto inicial } \\
\text { curva }\end{array}$} & \multirow{2}{*}{$\begin{array}{c}\begin{array}{c}\text { Punto final } \\
\text { curva }\end{array} \\
\mathrm{mPa} \\
\end{array}$} & \multirow{2}{*}{$\frac{\Delta \mathrm{T}}{\mathrm{mPa}}$} \\
\hline & & & & \\
\hline 1 & $\begin{array}{l}\text { Plástico con } \\
\text { tixotropía }\end{array}$ & 2842,1 & 527,4 & 2314,7 \\
\hline 2 & $\begin{array}{l}\text { Plástico con } \\
\text { tixotropía }\end{array}$ & 1992,4 & 615,3 & 1377,1 \\
\hline 3 & $\begin{array}{l}\text { Dilatante con } \\
\text { tixotropía }\end{array}$ & 2607,7 & 498,1 & 2109,4 \\
\hline 4 & $\begin{array}{l}\text { Plástico con } \\
\text { tixotropía }\end{array}$ & 1435,7 & 322,3 & 1113,4 \\
\hline 5 & $\begin{array}{l}\text { Plástico con } \\
\text { tixotropía }\end{array}$ & 2242,4 & 322,3 & 1920,1 \\
\hline 6 & $\begin{array}{l}\text { Plástico con } \\
\text { tixotropía }\end{array}$ & 1670,1 & 322,3 & 1347,8 \\
\hline 7 & $\begin{array}{l}\text { Plástico con } \\
\text { tixotropía }\end{array}$ & 1728,7 & 322,3 & 1406,4 \\
\hline 8 & $\begin{array}{l}\text { Plástico con } \\
\text { tixotropía }\end{array}$ & 2226,8 & 380,9 & 1845,9 \\
\hline 9 & $\begin{array}{l}\text { Plástico con } \\
\text { tixotropía }\end{array}$ & 2519,8 & 410,2 & 2109,6 \\
\hline 10 & $\begin{array}{l}\text { Plástico con } \\
\text { tixotropía }\end{array}$ & 2754,2 & 586,0 & 2168,2 \\
\hline 11 & $\begin{array}{l}\text { Plástico con } \\
\text { tixotropía }\end{array}$ & 3364,8 & 673,9 & 2690,9 \\
\hline
\end{tabular}

La tabla 8 es el resumen de los resultados de reología e indica el sistema reológico al que pertenece cada tratamiento; además, la diferencia entre los puntos inicial y final de las curvas, permite observar que el tratamiento 4 es más plástico por tener un valor menor, es decir, tiende a recuperar su estructura luego de ser sometida a la fuerza de deformación.

\section{Conclusiones}

- De acuerdo al análisis estadístico presentado en las tablas 2 y 3 se determinó que las materias primas que presentan similares características en punto de fusión, índice de yodo y contenido de sólidos grasos, son aceite de palma, palmiste y soya. La estearina de palma presenta diferencias en el contenido de sólidos grasos como se comprobó en la prueba t en la que se obtuvo un valor experimental de 32,315; en tanto que la t tabulada al $99 \%$ es de 9.92 . Por lo cual se aceptó la hipótesis alterna, que indica que la estearina ha cambiado en su composición química e influye en la mezcla para la fase oleosa.

- La evaluación sensorial determinó que de las once muestras de margarina, diez alcanzaron un buen grado de aceptación. En tanto que una fue catalogada como defectuosa por perder su consistencia al momento de ser usada en panificación; ésta fue producida bajo la condición de temperatura de emulsión elevada aproximadamente $52^{\circ} \mathrm{C}$.

- El análisis reológico de las margarinas, comprueba que se trata de sistemas tixotrópicos, como ya lo afirmó Bailey "las grasas tienen la estructura característica de un sólido plástico, pero, con frecuencia sufren fenómenos de tixotropía cuando se aplica sobre ellas trabajo mecánico" [3]. Tomando en cuenta los resultados de la tabla 7 , la muestra del tratamiento 4 presenta mayor estabilidad a la deformación ya que la variación entre los puntos iniciales y finales fue de $1113,4 \mathrm{mPa}$, un valor inferior a los demás tratamientos.

- Utilizando el microscopio de luz polarizada y mediante la comparación de las microfotografías experimentales con las del gráfico 3, se identificó algunas formas cristalinas, y se clasificó según su tamaño en posibles cristales $\beta$ tratamientos 6,9 y 11; posibles cristales $B^{\prime}$ tratamientos, $1,2,3,4,5$, 7,8 y 10.

- El análisis de calorimetría diferencial de barrido permitió establecer los tipos de cristales en las muestras. Tratamiento 3 sistemas cristalinos $B$; tratamiento 4 dos tipos de cristales $\beta^{\prime}$; tratamientos 1 , 6, 7 y 9 únicamente cristales $\beta^{\prime}$. Por lo tanto, las condiciones adecuadas para procesar margarina y obtener cristales $\beta^{\prime}$ son los tratamientos $4,6,7$ y 9 (Tabla 8); además, estos tratamientos cuentan con la aceptación mediante las pruebas de evaluación sensorial. 
Tabla 9. Parámetros óptimos de producción

\begin{tabular}{lcrrrr}
\hline \multirow{2}{*}{ PARÁMETROS } & \multirow{2}{*}{ Unidad } & \multicolumn{5}{c}{ Tratamientos } \\
\cline { 3 - 7 } & & \multicolumn{1}{c}{$\mathbf{4}$} & \multicolumn{1}{c}{$\mathbf{~}$} & \multicolumn{1}{c}{$\mathbf{7}$} & \multicolumn{1}{c}{$\mathbf{9}$} \\
\hline Caudal & 1670 & 1614 & 1670 & 1670 \\
Temperatura de emulsión & ${ }^{\circ} \mathrm{C}$ & 50,3 & 50,73 & 50,83 & 51,73 \\
Temperatura de enfriamiento & ${ }^{\circ} \mathrm{C}$ & 15,33 & 17,03 & 17,68 & 17,35 \\
Velocidad cristalizador 1 & $\mathrm{rpm}$ & 231 & 231 & 231 & 230 \\
Velocidad cristalizador 2 & $\mathrm{rpm}$ & 315 & 315 & 315 & 301 \\
Temperatura de empaque & ${ }^{\circ} \mathrm{C}$ & 25,88 & 27,0 & 27,15 & 27,4 \\
\hline
\end{tabular}

\section{Referencias}

1. Inec. "Censo poblacional". [en línea]. [consulta: 10 de mayo del 2009], Disponible en Web: www.inec.gov.ec/c/document_library/get_file?...\&name=DLFE-5137.pdf

2. Belitz, Hans-Dieter; Grosch, Werner. "Química de los alimentos". 2 ed., Zaragoza (España), Acribia, 1997 imp.

3. Bailey, Alton. "Aceites y grasas industriales". Traducido de la 2 ed. en inglés, Buenos Aires, Reverte, 1979.

4. Romo, Luis. "Coloideofísica, coloideoquímica: Fenómenos de superficie". 1 ed., Quito, UCE, Editorial Universitaria, 1981.

5. Gerstenberg Schröder. "Crystallization techonology Shortening-General Information". [en línea], Revisión Abril 2009, [Consulta: 9 de octubre del 2009], Disponible en web:http://www.gs-as.com/Art\%C3\%ADculos_de_investigaci\%C3\%B3n_y_desarrollo_.aspx?ID=1490

6. BDN Food Technology. "Margarinas". [en línea], Publicación 2001, [Consulta: 12 de noviembre del 2009], Disponible en web: http:// bdnhome.com/tecnologia/temas/margarinas.pdf

7. Garti, Nissim. Sato, Kiyotaka. "Crystallization processes in fats and lipid system". New York, Marcel Dekker cop. 2001

8. Bailey's. "Industrial oil \& fat products". 5 ed., Y.H.HUI. Technology and commerce International, 1996. Vol 3.

9. Palsgaard A/S. "Margarinas/ Shortenings líquidas \& bombeables: Principales ingredientes en dichos productos". [en línea], MOLBAK, Anders, Publicación 21 julio 2009, [Consulta: 28 agosto del 2009], Disponible en web: www.palsgaard.com

10. Kaletunç, Gönül. "Calorimetry in food processing: analysis and design of food system". 1 ed., USA, Wiley-Blackwell and the Institute of Food Technologists, 2009.

11. DANEC, S.A. "Procedimientos de ensayo para Analistas / Auxiliares". basados en los Métodos Analíticos de la AOCS y en NTE INEN, Ecuador, 1998,

12. Anzaldúa-Morales, Antonio. "La evaluación sensorial de los alimentos en la teoría y la práctica". Zaragoza (España), Acribia, 1998.

13. Flint, Olga. "Microscopia de los alimentos, Manual de métodos prácticos utilizando la microscopia óptica". Zaragoza (España), Acribia, 1996. 